\title{
Empirical Analysis of Context Free Grammars and Parse Trees
}

\author{
J.Sreedhar \\ Research Scholar \\ Department of CSE \\ JNTUK \\ Kakinada, India-533003
}

\author{
S.Viswanadha Raju, Ph.D \\ Professor -CSE \& HOD \\ JNTUHCEJ \\ JNTUH,Hyderabad \\ India-500072
}

\author{
A.Vinaya Babu, Ph.D \\ Professor-CSE\&Principal \\ JNTUH, \\ Hyderabad \\ India-500072
}

\begin{abstract}
This research explores the impact of Context Free Grammars (CFG) and Parse Trees for construction of a Telugu Language Sentences. Based on the CFG here we derived the derivations for the respective strings. Later we constructed the Parser Trees for the above said strings. Finally we analysed whether the string is ambiguous or unambiguous. Here for analysis we considered the Large Scale Open Source Telugu carpus.
\end{abstract}

\section{Keywords}

CFG, Parse Trees, Derivations

\section{INTRODUCTION}

The syntax of a language may be specified using a notation called context free grammar (CFG),. A context free grammar consists of terminals, non-terminals, a start symbol and production rules. The set of tokens are called the terminal symbols. These are the basic symbols from which strings are formed. Non terminals are the symbols which represent syntactic variables that denote sets of strings. They do not exist in the source program they only help in defining the language generated by the grammar. One of the non-terminals designated as the start symbol. We shall follow the convention of listing the production for the start symbol. The set of strings denoted by the start symbol is the language defined by the grammar. A production rule has a non-terminal symbol on the left hand side followed by an arrow and a sequence of symbols on the right side. This sequence of symbols may contain a combination of terminals and nonterminals[9,11,13].

The organization of this paper is as follows: Section II describes the CFG and its notations, Section III deals with derivations of CFG Grammar, Section IV explores the Parser Trees, Section V shows the acknowledgements and Section VI deals with conclusion followed by the references.

\section{CONTEXT FREE GRAMMARS}

It may have more than one production rule for the same non terminal. In that case, we can group their right hand side by using symbol | to separate the alternate right hand side. CFG, sometimes called a phrase structure grammar[2] plays a central role in the description of natural languages. In general a CFG $[10,12,17]$ is a set of recursive rewriting rules called productions that are used to generate patterns of strings and it consists of the following components:

- A finite set of terminal symbols $(\Sigma)$.

- A finite set of non-terminal symbols (NT).
- A finite set of productions $(\mathrm{P})$

- A start symbol (S).

Let $\mathrm{G}$ be a Context Free Grammar for which the production rules are:

$$
\begin{aligned}
& \mathrm{S}=>\mathrm{NP} \quad \mathrm{VP} \\
& \mathrm{NP}=>\text { Noun } \mid \text { Pronoun } \\
& \mathrm{VP}=>\text { Verb } \mid \text { VP PP } \epsilon \\
& \mathrm{PP} \Rightarrow \mathrm{NP} \text { PP }|\mathrm{VPPP}| \epsilon \\
& \text { Noun } \Rightarrow \mathrm{n} \\
& \text { Verb } \Rightarrow \mathrm{v} \\
& \text { Pronoun } \Rightarrow \text { pn }
\end{aligned}
$$

Fig. 1: Context Free Grammar

\section{DERIVATIONS}

Here Derivation provides a means for generating the sentences of a language. If one chooses the leftmost nonterminal in a given sentential form then it is called leftmost derivation. If one chooses the rightmost non-terminal in a given sentential form then it is called rightmost derivation. Derivation from $\mathrm{S}$ means generation of string $\mathrm{w}$ from $\mathrm{S}$. Any language construct can be defined by the CFG $[3,15,16]$. The above grammar generates different strings by providing many sentential forms as shown below.

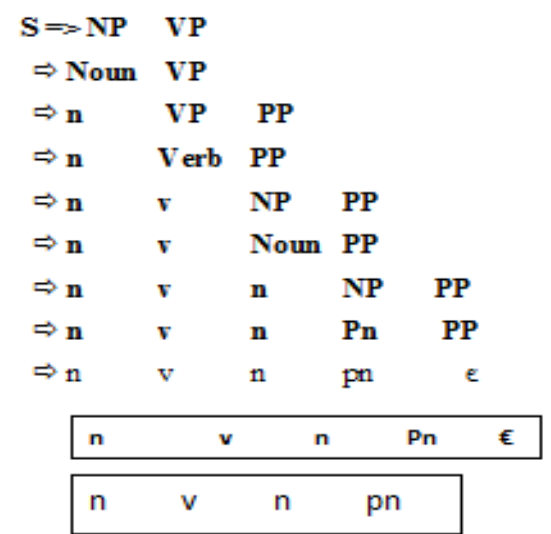

Fig 2: Derivation of " $n v \mathrm{n}$ pn" 
The start symbol of the above grammar is S. Any grammar contains terminals and non-terminals. The non-terminal symbol occurs at the left hand side. These are the symbols which need to be expanded.The non-terminals are replaced by the terminals which it derives.

The above string is derived from S step by step as follows:

- $\quad$ First the nonterminal NP present at the left side is replaced by its substring noun.

- Then it is substituted by its substring $n$.

- Then VP is substituted by its substring VP PP.

- Then again VP is substituted by its substring Verb.

- Then that Verb is substituted by its substring $\mathrm{v}$.

- Then PP is substituted by its substring NP PP.

- Then again NP is substituted by its substring Noun, and then Noun is substituted by its substring $n$.

- Then again PP is substituted by its substring NP PP.

- Then again NP is substituted by its substring Pronoun.

- $\quad$ Finally, PP is substitued by its substring $€$.

- So that, finally we obtain the string.

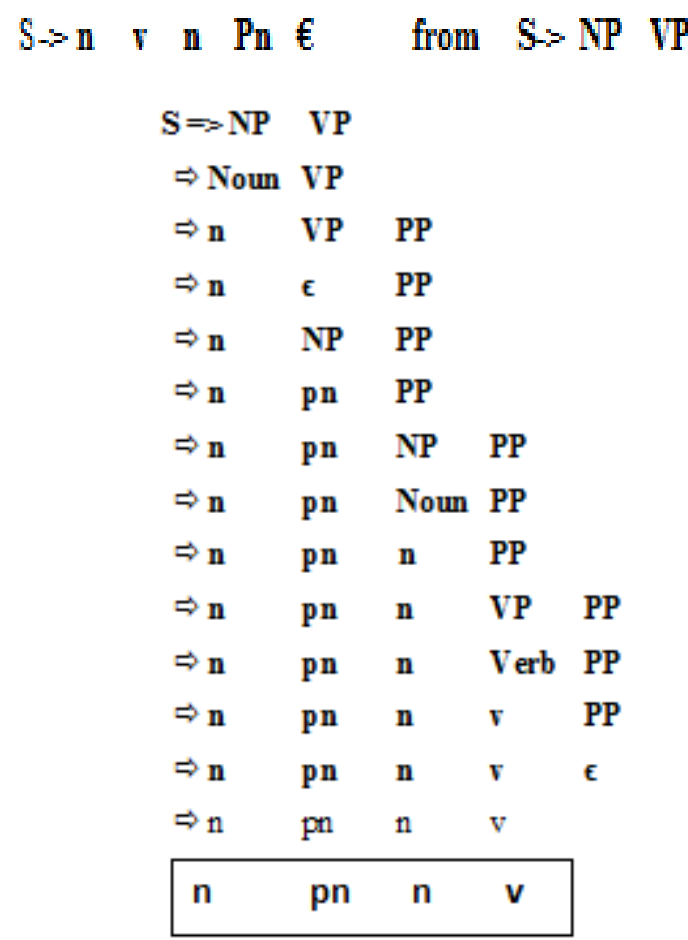

Fig 3: Derivation of " $n$ pn $n$ v"

The above string is derived from S step by step as follows:

- The non-terminal NP present at the left side is replaced by its substring noun.

- Then it is substituted by its substring $\mathrm{n}$.

- Then VP is substituted by its substring VP PP.

- Then again VP is substituted by its substring $€$.

- $€$ means null value, so we can just eliminate it.

- Then PP is substituted by its substring NP PP.
- Then NP is substituted by its substring pronoun (pn).

- Then again PP is substituted by its substring NP PP.

- Then again NP is substituted by its substring Noun, and then Noun is substituted by its substring $n$.

- Then again PP is substituted by its substring VP PP.

- Then again VP is substituted by its substring Verb ,and then Verb is substituted by one of the substring v.

- Finally, PP is substituted by its substring $€$.

- $€$ means null value, so we can just eliminate it.

- So that, finally we obtain the string

\section{$S>n$ pn $n \vee$ from $S>N P$ PP}

\section{PARSE TREES}

A parse tree[1,4,5] is an equivalent form of showing a derivation which represents a derivation graphically or pictorially. A parse-tree is an internal structure, created by the compiler or interpreter while parsing some language construction. Parsing is also known as 'syntax analysis'.

A parse tree for a grammar $\mathrm{G}$ is a tree where

- the root is the start symbol for $\mathrm{G}$

- the interior nodes are the non-terminals of $\mathrm{G}$

- the leaf nodes are the terminal symbols of G.

- the children of a node T (from left to right) correspond to the symbols on the right hand side of some production for $\mathrm{T}$ in $\mathrm{G}$.

Every terminal string generated by a grammar has a corresponding parse tree; every valid parse tree represents a string generated by the grammar (called the yield of the parse tree).

Example Parse Trees for NLP:

Consider the below grammar, implementing the parse tree for the strings generated by this grammar.

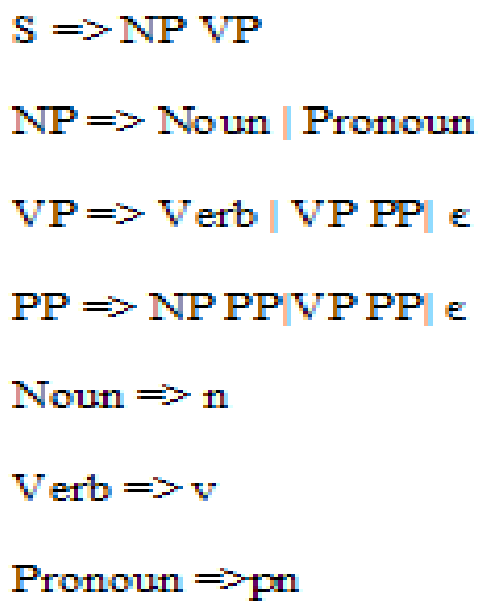

Fig. 4: Context Free Grammar 
1) This grammar generates the string $n \vee n p n$. The parse tree for this string using CFG is as following steps.

2) Create a root labeled with $S$.

3) For each sentential form $\alpha_{i}$ in the derivation, $i \geq 2$, construct a parse tree whose yield is $\alpha_{i}$, We can use induction for constructing the for $\alpha_{i}$, given the tree for $\alpha_{i-1}$ as given below:

a. The tree for $\alpha_{1}=\mathrm{S}$ is a single node labeled S.

$\mathrm{b}$.Let $\alpha_{\mathrm{i}-1}=\mathrm{X}_{1} \mathrm{X}_{2} \ldots . \mathrm{X}_{\mathrm{r}}$ and $\alpha_{\mathrm{i}}$ is derived from $\alpha_{\mathrm{i}-1}$ by replacing $X_{j}$ by $\beta=Y_{1} Y_{2} \ldots . . Y_{k}$.

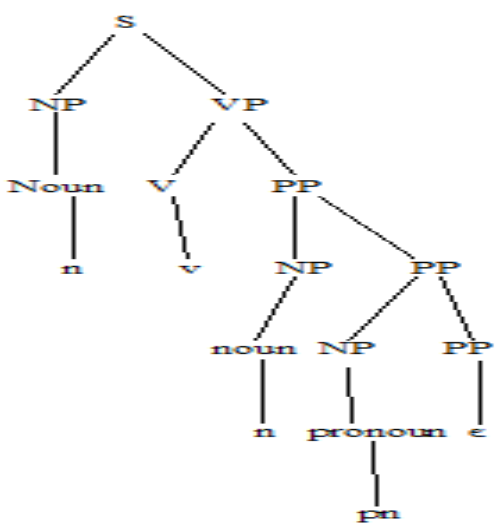

Fig 5: Parse Tree for "nvnpn"

$\mathrm{S}$ is a start symbol which derives NP VP, NP is a non-terminal which is substituted by noun and it is in turn substituted by the terminal $\mathrm{n}$.

Now VP derives VP PP, PP with NP PP. NP is substituted by noun and with $\mathrm{n}$.

Similarly PP derives NP PP and NP with the terminal pn. Finally, we obtain the string n v n pn.

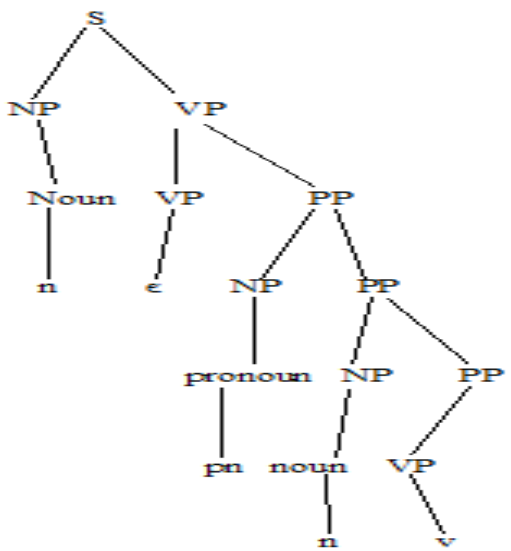

Fig 6: Parse Tree for "npnnv"

$\mathrm{S}$ is the start symbol for the above grammar which derives NP PP. NP is reduced to noun and inturn by $n$.

VP derives VP PP and PP to NP PP. Now NP is reduced to pronoun and to the terminal pn. Next PP is substituted by NP PP where NP to noun and PP to VP. Finally, we obtain the string $\mathrm{n}$ pn $\mathrm{n} \mathrm{v}$.

\section{ACKNOWLEDGMENTS}

We are very thankful to all the esteemed authors in a reference list, to make this research article in a better shape and in right direction

\section{CONCLUSION}

Here we described about the Context Free Grammars, Derivations and Parse Trees. We observed the ambiguity between the Telugu Language Sentences.

\section{REFERENCES}

[1] Aho, A.V., and Johnson, S. C. [1974]. "LR parsing," Computing Surveys 6:2,99-124.

[2] Aho, A.V., and Johnson, S. C. , and Ullman, J. D. [1975]. "Deterministic parsing of ambiguous grammars," Comm. ACM 18:8, 441-452.

[3] Aho, A.V., and Peterson, T.G, [1972]. "A minimum distance error correcting parser for context-free languages," SIAM J. Computing 1:4, 305-312.

[4] Aho, A.V., and Ullman, J. D. [1972b]. The Theory of Parsing Translation and Compiling, Vol. I:Parsing, Prentice-Hall, Englewood Cliffs, N. J.

[5] Aho, A.V., and Ullman, J. D. [1972c]. "Optimization of LR(k) parsers," J. Computer and systems Sciences 6:6, 573-602.

[6] Aho, A.V., and Ullman, J. D. [1972a]. The Theory of Parsing, Translation and Compiling, Vol. II: Compiling, Prentice- Hall, Englewood Cliffs, N. J.

[7] Aho, A.V., and Ullman, J. D. [1972b]. " A technique for speeding up LR(k) parsers.” SIAM J. Computing 2:2, 106-127.

[8] Anderson, J. P. [1964]. "A note on some compiling algorithms," comn. ACM 7:3, 149-153.

[9] Anderson, T., Eve, J., and Horning, J. J.[1973]. “ Efficient LR(1) paresers,” Acta Informatica 2:1, 12-39.

[10] Backhouse, R.C. [1976]. "An alternative approach to the improvement of LR parsers," Acta Informatica 6:3, 277 296.

[11] Bar Hillel, Y., Perles, M., and Shamir, E. [1961]. "On formal properties of simple phrase structure grammers," Z. Phonetik, Sprachwissenschaft und Kommunikationsforschung 14, pp. 143-172.

[12] Barnard, D. T. [1975]. "A survey of syntax error handling techniques," Computer Science Reaserch Group, Univ. of Toronto, Toronto, Ont., Canada.

[13] Birman, A., and Ullman, J.D. [1973]. "Parsing algorithms with backtrack," Information and Control 23:1, 1-34.

[14] Bochmann, G. V. [1976]. "Semantic evaluation from left to right," Comm. ACM 19:2, 55-62.

[15] Brzozowiski, J. A. [1964]. "Derivatives of regular expressions," J. ACM 11:4, 481-488.

[16] Cheatham, T. E. Jr., and Sattley, K. [1964]. "Syntax directed compiling," Proc. AFIPS 1964 Spring Joint Computer Conf. Spartan Books, Baltimore Md., 31-57.

[17] Chomsky, N. [1959]. "On Certain formal properties of grammers," Information and Control 2:2, 137-167. 